\title{
Improved high error tolerant data transmission algorithm for Vanet based on path criterion
}

\author{
Li Zhu ${ }^{1,2}$, Jianwu $\mathrm{Li}^{3, *}$ \\ ${ }^{1}$ China Transport Telecommunications \& Information Center, Beijing 100011, China \\ ${ }^{2}$ People's Public Security University of China, Beijing 100038, China \\ ${ }^{3}$ Cyber Space Institute of China Center for Informatica Industry Development, Beijing 100846, China \\ *Corresponding author
}

Keywords: time headway; transmission range; vehicle safety; Vehicular ad hoc network (VANETs)

\begin{abstract}
An Adaptive-Set-Range Rate (ASRR) algorithm, for adaptive set of the transmission range of BSM and the generation rate of data packet, is proposed in this Paper for reason that the safe application of VANETs requiring a periodic interaction of Basic Safety Message (BSM) by vehicles. Considering the safety of vehicles, the proposed ASRR algorithm is used to optimize the generation rate and transmission range of BSM, so as to maximize the utilization of the control channel. In the proposed ASRR algorithm, the time headway is considered as the performance indicators for safety of running vehicles, for calculating the generation rate of data packet, and the functional relationship between the time headway and the generation rate of data packet is set accordingly. Afterwards, the generation rate of data packet is used to calculate the transmission range, considering the consistency with transmission range of neighbor areas. The results of simulation show that, the proposed ASRR algorithm can significantly promote the packet success rate and the channel busy percentage as compared with FRT (fixed transmission range and generation rate of data packet). Thus, the Vehicular ad hoc network (VANETs) is considered as an important part of next generation of automotive system.
\end{abstract}

\section{Introduction}

The Vehicular ad hoc network (VANETs) provides a platform for Vehicle-to-Vehicle (V2V) communications. Through V2V communications, drivers are able to know the information about other vehicles and road conditions in real time, and to take prior actions to reduce and avoid accidents [1-2]. For safety message application, VANETs requires a periodic interaction of safety message, called Basic Safety Message (BSM), by vehicles. In the safety message application, the transmission range of BSM is $150-300 \mathrm{~m}$, with a generation rate of about $1-10 \mathrm{~Hz}$ [3]. The larger the transmission range, the farther the corresponding safety message is transmitted, the higher the safety. However, always using large transmission makes it easy to interfere with other vehicles and causes congestion, leading to packet dropout in areas of congested vehicles [4]. Similarly, the higher the generation rate of packet, the more the packet received from neighbor vehicles by the vehicle, the larger the amount of information obtained, and the safer the driving. However, transmission of a large amount of packets in channel would also cause congestion, lead to packet dropout. Especially in areas of congested vehicles, the congestion is more seriously and the packet dropout is more.

\section{ASRR Algorithm}

\subsection{Control of packet generation rate}

The time headway is considered as the indicator for controlling the packet generation rate. In a given traffic scene, the time headway $T_{H}$ is used to reflect the vehicle safety conditions [6]. Time headway means the interval between the time $t_{1}$ when the vehicle forward passes through a specific 
position and the time $t_{2}$ when the vehicle behind closely passes through the same position, in which $T_{H}=t_{2}-t_{1}$. For vehicles $m^{m}$ and ${ }^{n}$ driving from west to east, with vehicle $m$ forward and vehicle $^{n}$ behind closely, we assume that vehicle ${ }^{m}$ passes through a specific position (assumed to be the position of the house in figure), then the time headway of vehicle ${ }^{n}$ is the time passing through the house by it. The time headway may be calculated through the distance between frame head of two vehicles and the speed of vehicles behind, as shown in Formula (1).

$$
T_{H}(n)=\frac{d}{v_{n}}
$$

In which, $T_{H}(n)$ means the time headway of vehicle ${ }^{n}, d$ means the distance between frame head of two vehicles and $v_{n}$ means the speed of vehicle ${ }^{n}$.

As one of the important safety parameters, the time headway can be used to prevent collision by drivers if obtained in real time. The time headway reflects the spatial distance between two vehicles. The higher the time headway, the safer the road is. In case of an emergency, 1.5-2.0s of time headway can prevent collision.

In this Paper, we assume that the accurate position of each vehicle can be obtained through Global Positioning System (GPS). The position of vehicle and current driving speed are the part of BSM packet.

In fact, each vehicle is correlated with two time headways. The vehicle $n$ involves $T_{H}(n)$ and $T_{H}(k)$, in which $T_{H}(n)$ is calculated for vehicles $n$ and $k$ with Formula (1) respectively. The vehicle $^{n}$ extracts the $T_{H}(k)$ value from BSM packet sent by vehicle $k$. Both the two time headways $T_{H}(n) T_{H}(k)$ affect the safety of vehicle ${ }^{n}$. Thus, such two parameters should be considered when setting the packet generation rate of vehicle ${ }^{n}$. In the proposed algorithm for controlling the packet generation rate, considering the safety of vehicle ${ }^{n}$, minimum values of $T_{H}(n)$ and $T_{H}(k)$ are considered as the final time headway $T_{H}^{F}(n)$ of vehicle ${ }^{n}$, as shown in Formula (2), and $T_{H}^{F}(n)$ is used to calculate the packet generation rate.

$$
T_{H}^{F}(n)=\min \left(T_{H}(k), T_{H}(n)\right)
$$

The time headway provides a performance indicator for safe space between vehicles. The time headway is greater than the safe time headway (1.5s-2s) indicates that the road is safe and the BSM generation rate can be reduced. The time headway is $1.5 \mathrm{~s}$ smaller than the safe time headway, indicates the packet is generated at maximum rate. If within the range of 1.5-1.0, the packet generation rate reduces exponentially with the time headway. If beyond 10s, the packet is generated at minimum rate. Thus, the BSM generation rate can be calculated with Formula (3).

$$
R=\left\{\begin{array}{c}
10, T_{H}<1.5 \\
\mathrm{e}^{-0.2708 T_{H}+2.7092}, 1.5 \leq T_{H} \leq 10 \\
1, T_{H}>10
\end{array}\right.
$$

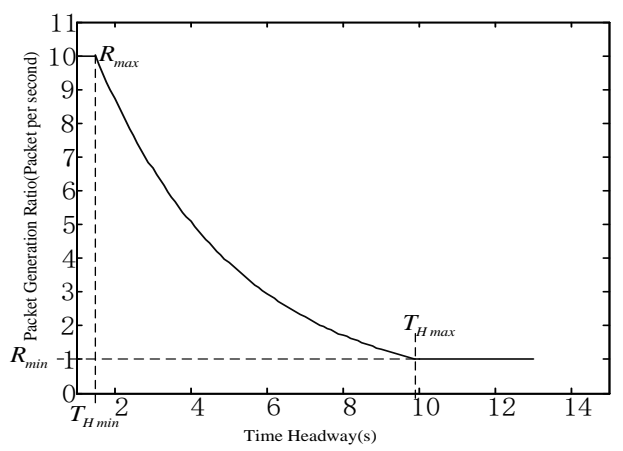

Fig.1 BSM Generation Rate at Different Time Headways

The curve of packet generation rate with the time headway varies is shown in Fig.1. In which, 
the minimum time headway $T_{H \text { min }}$ corresponds to the maximum BSM generation rate $R_{\max }$. As shown in Fig.3, between the minimum time headway $T_{H \min }$ and maximum time headway $T_{H \max }\left(T_{H \min }, T_{H \max }\right)$, BSM packet generation rate reduces exponentially. If the time headway is beyond the interval $^{\left(T_{H \min }, T_{H \max }\right)}$, the BSM packet generation rate is fixed. The exponential curve varies with the change of time headway, promoting road safety. And with increased time headway, the BSM packet generation rate reduces accordingly.

\subsection{Selection of transmission range}

Each vehicle estimates its transmission range according to the network load, and such estimated transmission range should be consistent with that selected by neighbor nodes.

The network load $N_{L}$ is defined as the average channel burden within the carrier sense range of vehicle. Within CCHI, the network load is correlated with the packet size $P_{s}$, the packet generation rate $R$, the vehicle density $V_{D}$, the carrier sense range $C_{S R}$ and total CCHI per second $T_{C C H}$, as shown in Formula (4).

$$
N_{L}=\frac{P_{s}^{*} R * V_{D} * 2 C_{S R}}{T_{C C H I}}
$$

The BSM packet has a size of 344bytes, including 80 bytes of information entity, 200 bytes of safety overhead and 64 bytes of MAC overhead. The carrier sense range of a vehicle is $x$ times the transmission range $T_{R}$, in which the value of $x$ depends on the threshold of carrier sense. In following simulation and analysis, we assume $x=1.46, T_{\text {ССнг }}=460 \mathrm{~ms}$ and the network load $N_{L}$ equals to $6 \mathrm{Mbps}$, the data rate for transmission of safety message. Thus, Formula (4) can be converted into Formula (5) as follows:

$$
T_{R}=\frac{343.5}{R \cdot V_{D}}
$$

As shown in Formula (5), the vehicle density $V_{D}$ needs to be estimated for calculation of transmission range ${ }^{T_{R}}$. Next, we analyze the estimation of vehicle density.

In fact, the estimated density of all vehicles in a certain area should be the same. However, the estimated density of each vehicle may not be consistent. For this, we take the average of density $\hat{V}_{D}(n)$ of vehicle $n$ and the density $\hat{V}_{D}(i)$ of neighbor vehicle as the final density $V_{D}$ of vehicle $n$, in which $^{i}$ is the neighbor of vehicle ${ }^{n}$, as shown in Formula (6)

$$
V_{D}=(\hat{V}_{D}(n)+\underbrace{\hat{V}_{D}(1)+\hat{V}_{D}(2)+\cdots}_{M \text { neighbors }}) /(1+M)
$$

In which, $M$ means $M$ neighbor vehicles of vehicle ${ }^{n}$, and $\hat{V}_{D}(1)$ means the estimated density of neighbor vehicle 1

The vehicle density $V_{D}$ and the packet generation rate $R$ are substituted in Formula (5) to estimate its transmission range ${ }^{T_{R}}$. Since each vehicle has different packet generation rate $R$, the estimated density of each vehicle is different too. However, vehicles with large transmission range $T_{R}$ may interfere with the transmission of safety message by vehicles with small transmission range $^{T_{R}}$. Therefore, in order to prevent inconsistent transmission range between vehicles, the transmission range of neighbor vehicles should be kept in consistency. Hence, the transmission range of the area should equal to the average of estimated transmission range of the vehicle and the estimated transmission range of neighbor vehicle, so as to keep consistent transmission range of all vehicles in a local area, as shown in Formula (7). 


$$
T_{R}=(\hat{T}_{R}(n)+\underbrace{\hat{T}_{R}(1)+\hat{T}_{R}(n)+\cdots}_{M \text { neighbors }}) /(1+M)
$$

In which, $\hat{T}_{R}(n)$ means the estimated transmission range of vehicle $n$ through Formula (5) and $\hat{T}_{R}(1)$ means the estimated transmission range of neighbor vehicle 1.

Vehicles can calculate the packet generation rate Rand transmission range $T_{R}$ through the algorithms for controlling the packet generation rate and for selecting the transmission range, and send data packets at parameters Rand ${ }^{T_{R}}$.

\section{Performance analysis}

\subsection{Simulation parameters and models}

The performance of the proposed ASRR algorithm is analyzed in this section. The simulation model based on OPNET Modeler 16.0 is selected and an expressway with length of 5km and two-way six lanes is used as the simulated scene. The traffic model of vehicles used in simulation is as follows: vehicle spacing is subject to an exponential distribution at low vehicle density (60 vehicles $/ \mathrm{km})$ and a normal distribution at high vehicle density $(120,180$ vehicles $/ \mathrm{km})$. The average speed of vehicles executed in the stimulation is selected at low, medium and high range, $40 \mathrm{~km} / \mathrm{h}$, $70 \mathrm{~km} / \mathrm{h}$ and $100 \mathrm{~km} / \mathrm{h}$, respectively. The vehicle speed is subject to a normal distribution at variance of $10 \%$ of the average speed. Moreover, Nakagami fading model is used, with index $m=3$. Each of stimulations takes 300s and specific parameters are as shown in Table 1.

Table 1 Important simulation parameters

\begin{tabular}{ll}
\hline Road Length and Number of Lanes & $5 \mathrm{~km} 6$ lanes (3 per way) \\
\hline Vehicle density & $60,120,180 \mathrm{vehicles} / \mathrm{km}$ \\
Vehicle speed & 40,70 and $\mathrm{km} / \mathrm{h}$ \\
BSM packet size & $344 \mathrm{bytes}$ \\
Data rate and frequency & $6 \mathrm{Mbps}$ and $5.9 \mathrm{GHz}$ \\
MAC/PHY & IEEE 802.11p \\
Propagation model & Nakagami fading $(\mathrm{m}=3)$ \\
Carrier sense threshold & $-94 \mathrm{dBm}$ \\
Background noise & $-99 \mathrm{dBm}$ \\
\hline
\end{tabular}

In order to better evaluate the performance of the proposed algorithm, packet success rate, packet generation rate and channel busy percentage are used. In which the channel busy percentage is defined as the percentage of channel busy time in the time sensed and the channel is considered as busy if the power received is greater than the carrier sense threshold.

\subsection{Numerical analysis after stimulation}

\subsubsection{BSM packet generation rate}

Fig.2 describes the curve of the BSM packet generation rate with the changes of vehicle speed and vehicle density. We can see from Fig.6 that, the BSM packet generation rate rises up as the average vehicle speed increases, as increased average vehicle speed leads to reduced time headway, thus promoting the BSM packet generation rate. Similarly, increased vehicle density shortens the average spacing between vehicles, which reduces the time headway and promotes the BSM packet generation rate. In addition, according to the traffic flow theory, the vehicle speed reduces as the vehicle density increases. Therefore, vehicles should be running at low speed at high density, for example, 180vehicles/km. Hence, there is no significant difference between the promoted BSM packet generation rate at average speed of $100 \mathrm{~km} / \mathrm{h}$ and $70 \mathrm{~km} / \mathrm{h}$. 


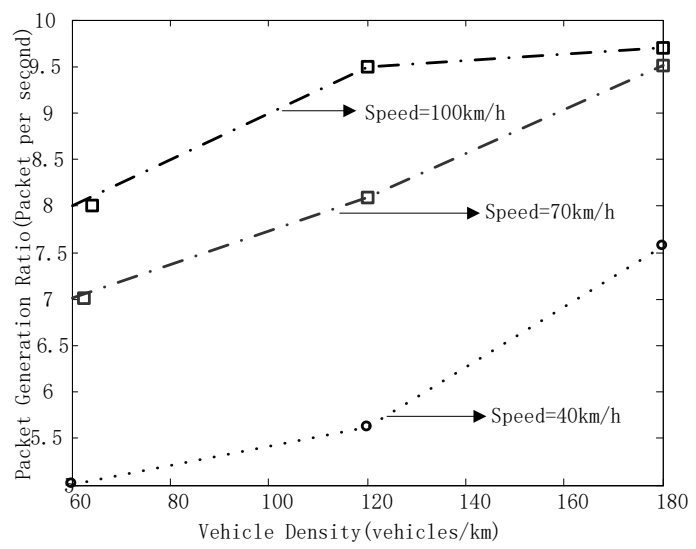

Fig.2 Curve of ASRR Packet Generation Rate with Changes of Vehicle Density

\subsubsection{Packet success rate}

The proposed ASRR algorithm is compared with the algorithm based on fixed rate transmission (FRT) in this experiment. The FRT algorithm is based on standard protocol IEEE 802.11p, and uses a fixed BSM packet generation rate at 10packets/second and fixed transmission range at $300 \mathrm{~m}$. The results of stimulation are as shown in Fig.5.

\section{Conclusions}

Most of safe applications of VANETs rely on reliable periodical transmission of basic safety message (BSM). However, due to changing vehicle density and vehicle speed, the fixed transmission parameters such as BSM transmission range and generation rate, may lead to data packet collision and reduce the utilization of control channel. Therefore, the transmission parameters should be adaptive to the changes in safe application of VANETs other than fixed. Considering the vehicle safety and maximizing the utilization of control channel, an Adaptive-Set-Range Rate (ASRR) algorithm, for adaptive set of the transmission range and the packet generation rate, is proposed in this Paper. Firstly, the time headway is measured and used as one of performance indicators for vehicle safety, and BSM generation rate is produced based on the time headway. Then the BSM generation rate, vehicle density and network load are used to calculate the transmission range, to make BSM generation rate and transmission range adaptively vary with vehicle density. The results of stimulation show that in a scene of changing vehicle density and vehicle speed, the proposed ASRR algorithm can be used to promote the success rate of safety message and the utilization of control channel.

\section{Acknowledgement}

This work was supported by key special project of National Key Research and Development Program (2017YFC0803900)

\section{References}

[1] Chai R, Yang B, Li L, et al. Clustering-based data transmission algorithms for VANET[C]// International Conference on Wireless Communications \& Signal Processing. IEEE, 2013:1-6.

[2] However, Terminals A “. Improved Data Transmission Scheme of Network Coding Based on Access Point Optimization in VANET [J]. Journal of Electrical \& Computer Engineering, 2014, 2014(11):1-5.

[3] Sou S I, Lee Y. Segcast: Segment-based broadcasting for delay tolerant data dissemination in intermittent VANETs[C]// International Conference on Information, Communications and Signal Processing. IEEE, 2016:1-5. 
[4] Nakamura M, Kitani T, Sun W, et al. A Method for Improving Data Delivery Efficiency in Delay Tolerant VANET with Scheduled Routes of Cars[C]// IEEE Conference on Consumer Communications and NETWORKING Conference. IEEE Press, 2010:1009-1013.

[5] Chai R, Ge X, Hu X, et al. Work in progress paper: Utility based clustering algorithm for VANET[C]// International Conference on Communications and NETWORKING in China. IEEE, 2015:187-190. 